\title{
Robust Tracking Control of a Nonholonomic Mobile Robot in the Presence of Disturbances*
}

\author{
Nobutaka WADA**, Masami SAEKI** and Haijun CHEN** $^{* *}$
}

\begin{abstract}
In this paper, we consider the tracking control problem of a nonholonomic mobile robot in the presence of bounded disturbances. We propose a method for modifying the control law of Jiang et al. (1997) so that the resulting control law guarantees global stability and the tracking error converges to a neighborhood about zero that can be made arbitrary small in the presence of disturbances. Further, we show that the tracking error converge to zero in the absence of disturbances. A numerical example is provided to show effectiveness of the method.
\end{abstract}

Key Words: Moving Robot, Nonlinear Control, Robust Control, Unknown Disturbance

\section{Introduction}

Several methodologies for tracking control of mobile robots with nonholonomic constraints ${ }^{(2)}$ have been developed (see e.g., Refs. (4), (6) and (7)). The common features of the methods are: 1) the time derivative of the Lyapunov function used to derive the nonlinear control law is negative semi-definite and asymptotic stability is shown by using Barbălat's lemma ${ }^{(8)}$,2) the methods only deal with the cases where disturbances do not exist. There exist several ways for modifying the nonlinear control law so that the boundedness of the state trajectories is guaranteed against disturbances ${ }^{(8),(9)}$. However, these methods only treat the cases where the time derivative of the Lyapunov function is negative definite. Hence it is unclear whether the methods are effective to the case where the time derivative of the Lyapunov function is only negative semi-definite or not. On the other hand, in Ref. (3), it is shown that exploiting the damped dynamic oscillator makes it possible to construct a Lyapunov function whose time derivative is negative definite. However, the existence of the damped dynamic oscillator prohibits that the tracking errors converge to zero in the absence of disturbances and tends to deteriorate the initial response.

In this paper, we propose a method for modifying the control law of Ref. (6) so that the resulting control law guarantees global stability and the tracking error con-

\footnotetext{
* Received 20th August, 2003 (No. 03-4106)

** Dept. of Mechanical System Engineering, Hiroshima University, 1-4-1 Kagamiyama, Higashi-Hiroshima, Hiroshima 739-8527, Japan.

E-mail: nwada@mec.hiroshima-u.ac.jp
}

verges to a neighborhood about zero that can be made arbitrary small in the presence of bounded disturbances. Further, we show that the control law guarantees that the tracking error converge to zero in the absence of the disturbances.

This paper is organized as follows. In section 2, we formulate the tracking control problem of the mobile robot. In section 3, we show a control method in the case where the bounded disturbances exist. In section 4, we provide a numerical example to show effectiveness of the method. Finally, in section 5, we summarize the paper.

Notations: A signal $v(t)$ defined on $[0, \infty)$ is said to belong to $\mathcal{L}_{\infty}$, i.e., $v \in \mathcal{L}_{\infty}$, if $\|v\|_{\mathcal{L}_{\infty}} \triangleq \sup _{t \in[0, \infty)}\|v\|_{2}<\infty$, where $\|v\|_{2} \triangleq\left(v^{T} v\right)^{1 / 2}$. The asymptotic $\mathcal{L}_{\infty}$ norm of $v(t)$ is defined by $\|v\|_{a} \triangleq \lim _{t \rightarrow \infty} \sup \|v\|_{2}$. A signal $v(t)$ defined on $[0, \infty)$ is said to belong to $\mathcal{L}_{1}$, i.e., $v \in \mathcal{L}_{1}$, if $\|v\|_{\mathcal{L}_{1}} \triangleq \int_{0}^{\infty}\|v\|_{2} d t<\infty$.

\section{Problem Formulation}

Let us consider a wheeled mobile robot depicted in Fig. 1. We assume that the dynamics of the robot can be described by the following differential equations ${ }^{(5)}$.

$$
\begin{aligned}
& M(q) \ddot{q}+G(q)+F(\dot{q})+\tau_{d}=B(q) \tau+A^{T}(q) \lambda \\
& A(q) \dot{q}=0
\end{aligned}
$$

where

$$
\begin{aligned}
& M(q) \triangleq\left[\begin{array}{lll}
m & 0 & 0 \\
0 & m & 0 \\
0 & 0 & J
\end{array}\right], \quad B(q) \triangleq \frac{1}{r}\left[\begin{array}{cc}
\cos \theta & \cos \theta \\
\sin \theta & \sin \theta \\
b & -b
\end{array}\right] \\
& A(q) \triangleq[\sin \theta-\cos \theta 0]
\end{aligned}
$$

and $m$ denotes the mass of the robot, $J$ denotes the moment of inertia of the robot about a vertical axis through $P$, 


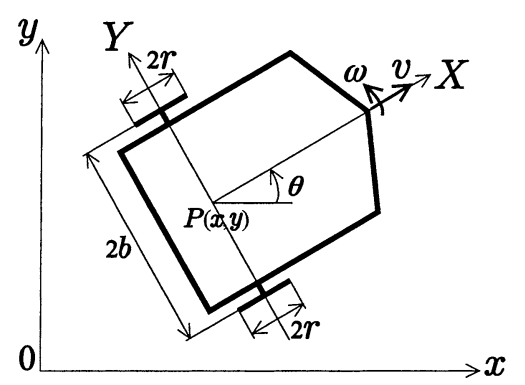

Fig. 1 Mobile robot model

$q \triangleq[x, y, \theta]^{T}, \tau \triangleq\left[\tau_{r}, \tau_{l}\right]^{T}$ denotes the input vector. $\lambda \in \mathcal{R}^{m}$ is the vector of constraint forces, and $\tau_{d} \in \mathcal{R}^{3}$ denotes a disturbance. $2 b$ is the width of the mobile robot and $r$ is the radius of the wheel. $M(q) \in \mathcal{R}^{3 \times 3}$ is the inertia matrix, $G(q) \in \mathcal{R}^{3 \times 1}$ is the gravity force, $F(\dot{q}) \in \mathcal{R}^{3 \times 1}$ is the surface friction, $B(q) \in \mathcal{R}^{3 \times 2}$ is the input transformation matrix, $A(q) \in \mathcal{R}^{1 \times 3}$ is the matrix associated with the nonholonomic constraints. We make the following assumption.

Assumption 1 There exists a positive scalar $\eta$ such that $\eta \geq\|d\|_{\mathcal{L}_{\infty}}$.

Then we introduce a full rank matrix $S(q) \in \mathcal{R}^{3 \times 2}$ which is made up by the smooth vectors fields and satisfies

$$
A(q) S(q)=0
$$

It is straightforward to verify that the following matrix satisfies Eq. (5).

$$
S(q) \triangleq\left[\begin{array}{cc}
\cos \theta & 0 \\
\sin \theta & 0 \\
0 & 1
\end{array}\right]
$$

From Eqs. (2) and (5), a kinematic model ${ }^{(1),(5)}$ of the mobile robot can be obtained as

$$
\dot{q}=S(q) v
$$

where $v(t) \triangleq[v, \omega]^{T} \in \mathcal{R}^{2}$.

Then, by differentiating Eq. (7), substituting the expression for $\ddot{q}$ into Eq. (1) and premultiplying by $S^{T}$, we have

$$
\bar{M} \dot{v}+\bar{V}_{m} v+\bar{G}+\bar{F}+\bar{d}=\bar{B} \tau
$$

where $\bar{M} \triangleq S^{T} M S, \bar{V}_{m} \triangleq S^{T} M \dot{S}, \bar{G} \triangleq S^{T} G, \bar{F} \triangleq S^{T} F, \bar{B} \triangleq$ $S^{T} B, \bar{d} \triangleq S^{T} \tau_{d}$. Then, by applying the following feedback control law,

$$
\tau=\bar{B}^{-1}\left[\bar{M} u+\bar{V}_{m} v+\bar{G}+\bar{F}\right]
$$

Equation (8) can be converted to

$$
\dot{v}=u+d
$$

where $d \triangleq-\bar{M}^{-1} \bar{d} \in R^{2}$ and $u \in R^{2}$ is a new input vector.

Then we introduce a reference robot described by

$$
\dot{q}_{r}=S\left(q_{r}\right) v_{r}
$$

where $q_{r} \triangleq\left[x_{r}, y_{r}, \theta_{r}\right]^{T}, v_{r} \triangleq\left[v_{r}, \omega_{r}\right]^{T}$, and the following error coordinates $^{(7)}$.

$$
\left[\begin{array}{l}
x_{e} \\
y_{e} \\
\theta_{e}
\end{array}\right]=\left[\begin{array}{ccc}
\cos \theta & \sin \theta & 0 \\
-\sin \theta & \cos \theta & 0 \\
0 & 0 & 1
\end{array}\right]\left[\begin{array}{l}
x_{r}-x \\
y_{r}-y \\
\theta_{r}-\theta
\end{array}\right]
$$

From Eqs. (7), (10)-(12), the error dynamics can be derived as

$$
\dot{\tilde{x}}=f(\tilde{x})+g(\tilde{x})[u+d]
$$

where

$$
f(\tilde{x}) \triangleq\left[\begin{array}{c}
\omega y_{e}-v+v_{r} \cos \theta_{e} \\
-\omega x_{e}+v_{r} \sin \theta_{e} \\
\omega_{r}-\omega \\
0 \\
0
\end{array}\right], g(\tilde{x}) \triangleq\left[\begin{array}{ll}
0 & 0 \\
0 & 0 \\
0 & 0 \\
1 & 0 \\
0 & 1
\end{array}\right]
$$

and $\tilde{x} \triangleq\left[x_{e}, y_{e}, \theta_{e}, v, \omega\right]^{T}$.

In this paper, we consider the following problem.

Problem 1 Consider the system (13). Find a feedback control law such that, for arbitrary initial tracking error, 1) all the state trajectories are globally bounded and the tracking errors $\left|x_{e}\right|,\left|y_{e}\right|,\left|\theta_{e}\right|$ converge to a neighborhood about zero that can be made arbitrary small in the presence of $d$, and 2) $\left|x_{e}\right|,\left|y_{e}\right|,\left|\theta_{e}\right|$ converge to zero in the case where there exists finite time $t_{d}$ such that $d(t)=0, \forall t \geq t_{d}$.

\section{Tracking Control of a Mobile Robot}

In this section, we first introduce a tracking control law of Ref. (6) that can be applied to the case where the disturbances do not exist. Then, we show a method for modifying the control law for the case where the disturbances exist.

\subsection{Tracking control in the absence of distur- bances}

By introducing the following new variables

$$
\begin{aligned}
\bar{x}_{e} \triangleq & x_{e}-c_{3} \omega y_{e}, \bar{v} \triangleq v-\alpha_{v}, \quad \bar{\omega} \triangleq \omega-\alpha_{\omega} \\
\alpha_{v} \triangleq & c_{4} \bar{x}_{e}+v_{r} \cos \theta_{e}-c_{3} \dot{\omega} y_{e} \\
& +c_{3} \omega\left[\omega x_{e}-v_{r} \sin \theta_{e}\right] \\
\alpha_{\omega} \triangleq & \omega_{r}+\gamma y_{e} v_{r} \frac{\sin \theta_{e}}{\theta_{e}}+c_{5} \gamma \theta_{e}
\end{aligned}
$$

where $c_{3}, c_{4}, c_{5}, \gamma>0$, the system (13) can be transformed into

$$
\dot{\mathbf{x}}=\mathbb{F}(\mathbf{x})+\mathbb{G}(\mathbf{x})[u+d]
$$

where

$$
\mathbb{F}(\mathbf{x}) \triangleq\left[\begin{array}{c}
\omega y_{e}-c_{4} \bar{x}_{e}-\bar{v} \\
-c_{3} \omega^{2} y_{e}-\omega \bar{x}_{e}+v_{r} \sin \theta_{e} \\
-c_{5} \gamma \theta_{e}-\gamma y_{e} v_{r} \frac{\sin \theta_{e}}{\theta_{e}}-\bar{\omega} \\
-\dot{\alpha}_{v} \\
-\dot{\alpha}_{\omega}
\end{array}\right], \quad \mathbb{G}(\mathbf{x}) \triangleq\left[\begin{array}{ll}
0 & 0 \\
0 & 0 \\
0 & 0 \\
1 & 0 \\
0 & 1
\end{array}\right]
$$

and $\mathbf{x}(t) \triangleq\left[\bar{x}_{e}, y_{e}, \theta_{e}, \bar{v}, \bar{\omega}\right]^{T}$. Then we apply the following feedback control law

$$
u(\mathbf{x})=\Upsilon(\mathbf{x}) \triangleq\left[\begin{array}{c}
\bar{x}_{e}+\dot{\alpha}_{v}-c_{6} \bar{v} \\
\frac{1}{\gamma} \theta_{e}+\dot{\alpha}_{\omega}-c_{7} \bar{\omega}
\end{array}\right]
$$


where $c_{6}, c_{7}>0$, and consider the following Lyapunov function candidate.

$$
V(\mathbf{x}) \triangleq \frac{1}{2} \bar{x}_{e}^{2}+\frac{1}{2} y_{e}^{2}+\frac{1}{2 \gamma} \theta_{e}^{2}+\frac{1}{2} \bar{v}^{2}+\frac{1}{2} \bar{\omega}^{2}
$$

Taking the time derivative of $V$ along the solution trajectories of Eqs. (18) and (19) with $d(t)=0, \forall t \geq 0$ yields

$$
\begin{aligned}
\dot{V}(\mathbf{x}) & =-c_{3} \omega^{2} y_{e}^{2}-c_{4} \bar{x}_{e}^{2}-c_{5} \theta_{e}^{2}-c_{6} \bar{v}^{2}-c_{7} \bar{\omega}^{2} \\
& \triangleq-W(\mathbf{x}) \leq 0
\end{aligned}
$$

Hence, in the case where $d(t)=0, \forall t \geq 0$, the control law (19) guarantees global stability in the sense of Lyapunov. Furthermore, although $W(\mathbf{x})$ is only positive semi-definite, it is shown in Ref. (6) that the control law also guarantees global asymptotic stability by applying Barbălat's lemma ${ }^{(8)}$.

Proposition $1{ }^{(6)}$ Consider the system (13), (19) with $d(t)=0, \forall t \geq 0$. Assume that $v_{r}, \dot{v}_{r}, \omega_{r}, \dot{\omega}_{r}$ and $\ddot{v}_{r}, \ddot{\omega}_{r}$ are bounded on $[0, \infty)$. Then all the state trajectories of the system (13), (19) are globally bounded. Furthermore, if $v_{r}(t)$ does not converge to zero, or if $v_{r}(t)$ tends to zero but $\omega_{r}(t)$ does not converge to zero, then

$$
\lim _{t \rightarrow \infty}\left[\left|x_{e}\right|+\left|y_{e}\right|+\left|\theta_{e}\right|+\left|\omega-\omega_{r}\right|+\left|v-v_{r}\right|\right]=0
$$

\subsection{Tracking control in the presence of distur- bances}

In the case of $d \neq 0$, the control law (19) may not guarantee not only the asymptotic property (22) but also boundedness of the state trajectory. To consider the case where the disturbance exists, we modify the control law (19) as follows.

$$
u(\mathbf{x})=\Upsilon(\mathbf{x})-\xi(\mathbf{x})
$$

where

$$
\xi(\mathbf{x}) \triangleq\left[\begin{array}{l}
\eta \tanh \left(\kappa z_{1}\right) \\
\eta \tanh \left(\kappa z_{2}\right)
\end{array}\right], \quad z \triangleq\left(\frac{\partial V}{\partial \mathbf{x}} \mathbb{G}\right)^{T}
$$

and $\kappa$ is a positive scalar.

By taking the time derivative of $V(\mathbf{x})$ along the solution trajectories of the system (18), (23), we have

$$
\dot{V} \leq-W(\mathbf{x})+\frac{2 \eta}{\kappa}, \quad \forall \mathbf{x} \in \mathcal{R}^{5}
$$

The derivation of Eq. (24) is shown in Appendix A.

Since $\dot{V}<0, \forall \mathrm{x} \in \bar{\Omega}_{W} \triangleq\left\{\mathrm{x} \in \mathcal{R}^{5} \mid W(\mathrm{x})>2 \eta / \kappa\right\}, V$ monotonically decreases while $x \in \bar{\Omega}_{W}$, which implies that any trajectory $\mathbf{x}(t)$ starting from $\bar{\Omega}_{W}$ is bounded while $\mathrm{x} \in \bar{\Omega}_{W}$. Then $\mathrm{x}(t)$ enters $\Omega_{W} \triangleq\left\{\mathrm{x} \in \mathcal{R}^{5} \mid W(\mathbf{x}) \leq 2 \eta / \kappa\right\}$ at some time or remains in $\bar{\Omega}_{W}, \forall t \geq 0$. In the following, we first examine the behavior of $\mathbf{x}(t)$ after its arrival at $\Omega_{W}$ in the case where $\mathrm{x}(t)$ enters $\Omega_{W}$ (Case 1$)$. Then we consider the case where $\mathrm{x}(t)$ remains in $\bar{\Omega}_{W}, \forall t \geq 0$ (Case 2). Finally, we consider the case where the initial state value belongs to $\Omega_{W}$, namely, $\mathbf{x}(0) \in \Omega_{W}$ (Case 3).
- $\quad$ Case 1: $\mathbf{x}(t)$ enters $\Omega_{W}$

When $\mathrm{x}(t)$ enters $\Omega_{W}$, the following inequality holds.

$$
c_{3} \omega^{2} y_{e}^{2}+c_{4} \bar{x}_{e}^{2}+c_{5} \theta_{e}^{2}+c_{6} \bar{v}^{2}+c_{7} \bar{\omega}^{2} \leq \frac{2 \eta}{\kappa}, \mathbf{x} \in \Omega_{W}
$$

Inequality (25) implies that

$$
\begin{aligned}
& \left|\omega y_{e}\right| \leq \sqrt{\frac{2 \eta}{c_{3} \kappa}},\left|\bar{x}_{e}\right| \leq \sqrt{\frac{2 \eta}{c_{4} \kappa}},\left|\theta_{e}\right| \leq \sqrt{\frac{2 \eta}{c_{5} \kappa}} \\
& |\bar{v}| \leq \sqrt{\frac{2 \eta}{c_{6} \kappa}},|\bar{\omega}| \leq \sqrt{\frac{2 \eta}{c_{7} \kappa}}, \quad \forall x \in \Omega_{W}
\end{aligned}
$$

From (26), we can conclude that the state variables except for $y_{e}$ are bounded on $\Omega_{W}$. Then we examine the boundedness of $y_{e}(t)$ on $\Omega_{W}$. In the following, we assume that there exists a constant $v_{r \min }$ such that $0<v_{r \min } \leq v_{r}, \forall t \geq 0$. From Eqs. (15) and (17), we have

$$
\omega=\mathbb{A} y_{e}+\mathbb{B}
$$

where $\mathbb{A}(t) \triangleq \gamma v_{r} \frac{\sin \theta_{e}}{\theta_{e}}, \mathbb{B}(t) \triangleq \bar{\omega}+\omega_{r}+c_{5} \gamma \theta_{e}$. Then, by substituting (27) for the 1st inequality of (26), we have

$$
\left|\omega y_{e}\right|=\left|\left(\mathbb{A} y_{e}+\mathbb{B}\right) y_{e}\right| \leq \rho, \quad \forall x \in \Omega_{W}
$$

where $\rho \triangleq \sqrt{2 \eta / c_{3} \kappa}$. If we choose $c_{5}$ and $\kappa$ such that $\sqrt{2 \eta / c_{5} \kappa}<\pi$, then $\mathbb{A}(t)>0, \forall x \in \Omega_{W}$. In this case, from the inequality (28), the upper bound of $\left|y_{e}\right|$ can be obtained as

$$
\begin{aligned}
\left|y_{e}\right| & \leq \frac{1}{2}\left(\frac{|\mathbb{B}|}{\mathbb{A}}+\sqrt{\left(\frac{\mathbb{B}}{\mathbb{A}}\right)^{2}+\frac{4 \rho}{\mathbb{A}}}\right) \\
& \leq \frac{1}{2}\left(\Phi+\sqrt{\Phi^{2}+4 \rho \Psi}\right), \quad \forall x \in \Omega_{W}
\end{aligned}
$$

where

$$
\begin{aligned}
& \Psi \triangleq \frac{1}{\gamma} \frac{1}{v_{r \min }} \frac{\sqrt{\frac{2 \eta}{c_{5} \kappa}}}{\sin \left(\sqrt{\frac{2 \eta}{c_{5} \kappa}}\right)} \\
& \Phi \triangleq \Psi(\gamma, \epsilon)\left[\omega_{r \text { max }}+\sqrt{\frac{2 \eta}{\kappa}}\left(\frac{1}{\sqrt{c_{7}}}+\gamma \sqrt{c_{5}}\right)\right]
\end{aligned}
$$

and $\omega_{r \max }$ is a constant such that $\omega_{r \max } \geq\left|\omega_{r}\right|, \forall t \geq 0$. Note that $\Psi(\gamma, \epsilon)$ and $\Phi(\gamma, \epsilon)$ can be made arbitrary small by choosing large values as $\gamma$ and $\kappa$.

From Eq. (29), when $v_{r} \geq v_{r \min }>0, \forall t \geq 0$ and $\sqrt{2 \eta / c_{5} \kappa}<\pi,\left|y_{e}\right|$ is also bounded on $\Omega_{W}$. Therefore, $\mathbf{x}(t)$ is bounded whenever $\mathbf{x}(t) \in \Omega_{W}$ since $\Omega_{W} \in$ $\Omega_{\mathrm{x}}$, where $\Omega_{\mathrm{x}}$ is a bounded set defined by $\Omega_{\mathrm{x}} \triangleq$ $\left\{x \in \mathcal{R}^{5} \mid\right.$ from the 2 nd to the 5 th inequalities of (26), (29) $\}$ (see Fig. 2).

Since $\Omega_{W}$ is not an invariant set in general, $\mathbf{x}(t)$ may leave $\Omega_{W}$ at some time. Hence, in the following, we consider the behavior of $\mathbf{x}(t)$ after it leaves $\Omega_{W}$. To consider the case, we define a level set $L_{V}(\beta) \triangleq\left\{\mathbf{x} \in \mathcal{R}^{5} \mid V(\mathbf{x}) \leq \beta\right\}$ such that $\Omega_{\mathrm{x}} \in L_{V}(\beta)$. Such a positive scalar $\beta$ can be derived by substituting the right side of Eqs. (26) and (29) 


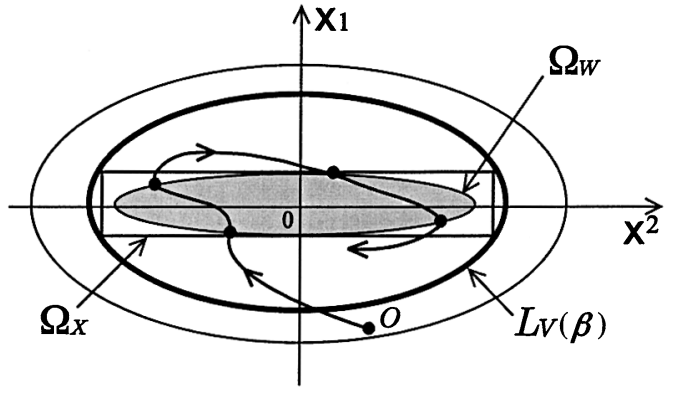

Fig. 2 Trajectory of the state variable $x(t)$

for Eq. (20) as follows.

$$
\beta \triangleq \frac{\eta}{c_{4} K}+\frac{1}{8}\left(\Phi+\sqrt{\Phi^{2}+4 \rho \Psi}\right)^{2}+\frac{\eta}{\gamma c_{5} K}+\frac{\eta}{c_{6} K}+\frac{\eta}{c_{7} K}
$$

The relationship between $\Omega_{\mathrm{x}}$ and $L_{V}(\beta)$ is shown in Fig. 2 . Note that $\beta$ defined in Eq. (30) can be made arbitrary small by choosing large values as $\gamma$ and $\kappa$. Since $\Omega_{\mathrm{x}} \in L_{V}(\beta)$ and $\dot{V}<0$ outside of $\Omega_{W}, \mathbf{x}(t)$ moves inside $L_{V}(\beta)$ and approaches $\Omega_{W}$ again. After that, although $\mathrm{x}(t)$ may reach and leave $\Omega_{W}$ repeatedly, $\mathbf{x}(t)$ stays in $L_{V}(\beta)$.

- Case 2: $\mathbf{x}(t)$ remains in $\bar{\Omega}_{W}, \forall t \geq 0$

When $\mathbf{x}(t) \in \bar{\Omega}_{W}, \forall t \geq 0, V$ monotonically decrease toward a constant $c>0$ since $\dot{V}<0$ and $\mathrm{x}=0 \in \Omega_{W}$. In this case, $\dot{V}$ converges to zero as $t \rightarrow \infty$, which implies that $\mathrm{x}(t)$ is globally bounded and converges to the boundary of $\Omega_{W}$ as $t \rightarrow \infty$. This implies that $\mathrm{x}(t)$ converges to $L_{V}(\beta)$ as $t \rightarrow \infty$ since $\Omega_{W} \in L_{V}(\beta)$.

- Case 3: $\mathbf{x}(0) \in \Omega_{W}$

Since $\Omega_{W} \in L_{V}(\beta), \mathbf{x}(0) \in \Omega_{W}$ implies $\mathbf{x}(0) \in L_{V}(\beta)$. Hence, in this case, it is clear that $\mathrm{x}(t) \in L_{V}(\beta), \forall t \geq 0$.

From the above discussion, we can conclude that any trajectory $\mathbf{x}(t)$ converges to the level set $L_{V}(\beta)$ as $t \rightarrow \infty$. Then, based on the result, we show that the upper bound of the asymptotic $\mathcal{L}_{\infty}$ norm of the error signals $x_{e}(t), y_{e}(t)$, $\theta_{e}(t)$ can be made arbitrary small by appropriately choosing the parameters of the control law. Since any state trajectory $\mathbf{x}(t)$ converges to $L_{V}(\beta)$ as $t \rightarrow \infty$, the following inequalities holds.

$$
\begin{aligned}
& \left\|\bar{x}_{e}\right\|_{a} \leq \sqrt{2 \beta},\left\|y_{e}\right\|_{a} \leq \sqrt{2 \beta},\left\|\theta_{e}\right\|_{a} \leq \sqrt{2 \beta \gamma} \\
& \|\bar{v}\|_{a} \leq \sqrt{2 \beta},\|\bar{\omega}\|_{a} \leq \sqrt{2 \beta}
\end{aligned}
$$

Therefore, by choosing a sufficiently small value as $\beta$, $\left\|y_{e}\right\|_{a}$ can be made arbitrary small. Next we consider the upper bound of $\left\|x_{e}\right\|_{a}$. From Eqs. (15), (17) and (31), we have

$$
\begin{aligned}
\left\|x_{e}\right\|_{a} \leq & \left\|\bar{x}_{e}\right\|_{a}+c_{3}\|\omega\|_{a}\left\|y_{e}\right\|_{a} \\
\leq & \sqrt{2 \beta}+c_{3} \sqrt{2 \beta}\left(\sqrt{2 \beta}+\left\|\omega_{r}\right\|_{a}\right. \\
& \left.+\gamma \sqrt{2 \beta}\left\|v_{r}\right\|_{a}+c_{5} \gamma \sqrt{2 \beta \gamma}\right)
\end{aligned}
$$

Hence, by choosing sufficiently small values as $\beta$ and $c_{3}$, the upper bound of $\left\|x_{e}\right\|_{a}$ can be made arbitrary small. (Al- though the right side of Eq. (32) becomes large when we choose a large value as $\gamma$, it can be made small by choosing a sufficiently small value as $c_{3}$.) Finally, we consider the upper bound of $\left\|\theta_{e}\right\|_{a}$. From Eq. (30), the following equality can be derived.

$$
\begin{aligned}
\beta \gamma= & \frac{\gamma \eta}{c_{4} \kappa}+\frac{1}{8}\left(\sqrt{\gamma} \Phi+\sqrt{\gamma \Phi^{2}+4 \rho \gamma \Psi}\right)^{2}+\frac{\eta}{c_{5} K}+\frac{\gamma \eta}{c_{6} \kappa} \\
& +\frac{\gamma \eta}{c_{7} K}
\end{aligned}
$$

From Eq. (33), it can be verified that the value $\beta \gamma$ can be made arbitrary small by choosing a sufficiently large value as $\kappa$, which implies that the upper bound of $\left\|\theta_{e}\right\|_{a}$ can be made arbitrary small.

Theorem 1 Consider the system (18), (23). Assume that Assumption 1 is satisfied. Further, assume that 1) there exist constants $x_{r \min }$ and $\omega_{r \max }$ such that $0<v_{r \min } \leq v_{r}$, $\forall t \geq 0$ and $\left.\left|\omega_{r}\right| \leq \omega_{r \max }, \forall t \geq 0,2\right) v_{r}, \dot{v}_{r}, \omega_{r}, \dot{\omega}_{r}$ are bounded on $[0, \infty)$ and 3$)$ the design parameters $\kappa$ and $c_{5}$ are chosen such that $\sqrt{2 \eta / c_{5} K}<\pi$. Then all the state trajectories of the system (18), (23) are globally bounded. Moreover, the upper bound of $\left\|x_{e}\right\|_{a},\left\|y_{e}\right\|_{a},\left\|\theta_{e}\right\|_{a}$ can be made arbitrary small by choosing large values as $\gamma, \kappa$ and a small value as $c_{3}$.

Furthermore, assume that there exists finite time $t_{d}$ such that $\tau_{d}(t)=0, \forall t \geq t_{d}$. Then, if $v_{r}(t)$ does not converge to zero, or if $v_{r}(t)$ tends to zero but $\omega_{r}(t)$ does not converge to zero, then

$$
\lim _{t \rightarrow \infty}\left[\left|x_{e}\right|+\left|y_{e}\right|+\left|\theta_{e}\right|+\left|\omega-\omega_{r}\right|+\left|v-v_{r}\right|\right]=0
$$

Proof) The first half of Theorem 1 has been already proven. The proof of the asymptotic property (34) is shown in Appendix B. $\square$

Note 1 The boundedness of the state trajectory of the system (18), (23) is guaranteed in the case where the conditions of Theorem 1 are satisfied. By using the result, it can be verified that the original state trajectory, namely, $\left[x_{e}, y_{e}, \theta_{e}, v, \omega\right]^{T}$, is also bounded (see Appendix D). However, unfortunately, this does not always imply boundedness of the control input $u(\mathrm{x})$. In order to guarantee boundedness of $u(\mathrm{x})$, several additional conditions, namely, $\ddot{v}_{r}, \ddot{\omega}_{r} \in \mathcal{L}_{\infty}$ and $\dot{\tau}_{d 3} \in \mathcal{L}_{\infty}$ are required (see Appendix $E$ ).

Note 2 The method of modifying the control law of this paper can be regarded as a so-called Lyapunov redesign technique $^{(8),(9)}$. However, the usual Lyapunov redesign technique ${ }^{(8),(9)}$ only treats the case where $W(\mathbf{x})$ is positive definite. Hence, since $W(\mathbf{x})$ of the control problem of this paper is positive semi-definite, the techniques used to prove stability in Refs. (8) and (9) can not be applied to the problem of this paper. Moreover, as opposed to the usual Lyapunov redesign technique, the term $\xi(\mathbf{x})$ introduced in Eq. (23) has two important properties, namely, 


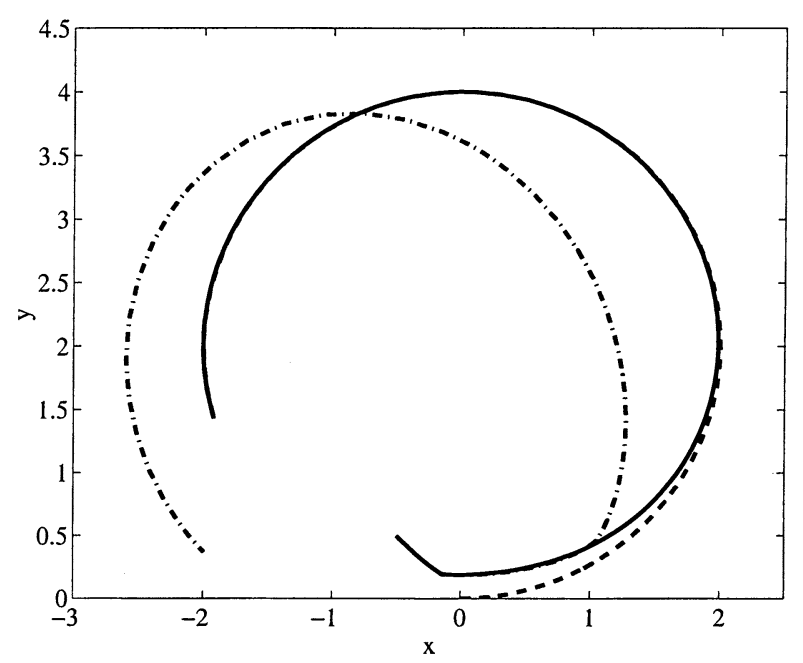

Fig. 3 Tracking trajectories (dashed: reference robot, solid: real robot with $\xi(\mathbf{x})$, dash-dot: real robot without $\xi(\mathbf{x})$ )

smoothness and boundedness. These properties enable us to prove that 1) $u(\mathbf{x})$ is bounded (see Appendix E) and 2) the asymptotic $\mathcal{L}_{\infty}$ norm of the signals $x_{e}, y_{e}, \theta_{e}$ can be made arbitrary small with keeping amplitude of $\xi_{i}(\mathbf{x})$ less than $\eta$.

\section{Numerical Example}

In this numerical example, physical parameters and design parameters are $m=1, J=1, r=1, b=1, G(q)=$ $0, \forall q \in \mathcal{R}^{3}, F(\dot{q})=0, \forall \dot{q} \in \mathcal{R}^{3}, c_{3}=c_{4}=c_{5}=c_{6}=c_{7}=1$, $\gamma=10, \kappa=100$. The bounded disturbance is

$$
\tau_{d}(t)= \begin{cases}{\left[\begin{array}{l}
3 \\
3 \\
3
\end{array}\right] \cos \left(\frac{\pi}{6} t\right),} & (0 \leq t \leq 3) \\
{\left[\begin{array}{l}
3 \\
3 \\
3
\end{array}\right],} & (3 \leq t)\end{cases}
$$

The positive scalar $\eta$ is chosen as $\eta=6$. The initial condition is $q_{r}(0)=[0,0,0]^{T}, q(0)=\left[-0.5,0.5,-\frac{\pi}{4}\right]^{T}$. Further, $v_{r}=1, \omega_{r}=0.5$.

In Figs. 3-5, it is shown that the state trajectory of the real robot with $\xi(x)$ is quite similar to that of the reference robot. On the other hand, the state trajectory of the real robot without $\xi(\mathbf{x})$ is deteriorated by the disturbance.

\section{Conclusion}

In this paper, we have considered the tracking control problem of a nonholonomic mobile robot in the presence of bounded disturbances. We have shown that, by using the proposed control law, all the state variables are globally bounded and the tracking error converges to a neighborhood about zero that can be made arbitrary small in the presence of disturbances. Furthermore, we have shown

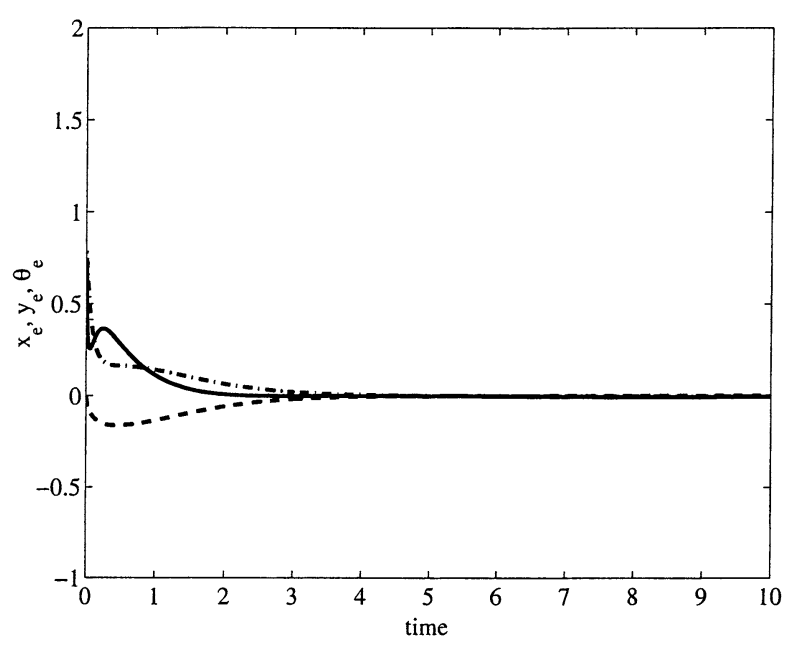

Fig. 4 Tracking errors with $\xi(\mathbf{x})$ (solid: $x_{e}$, dashed: $y_{e}$, dash$\left.\operatorname{dot}: \theta_{e}\right)$

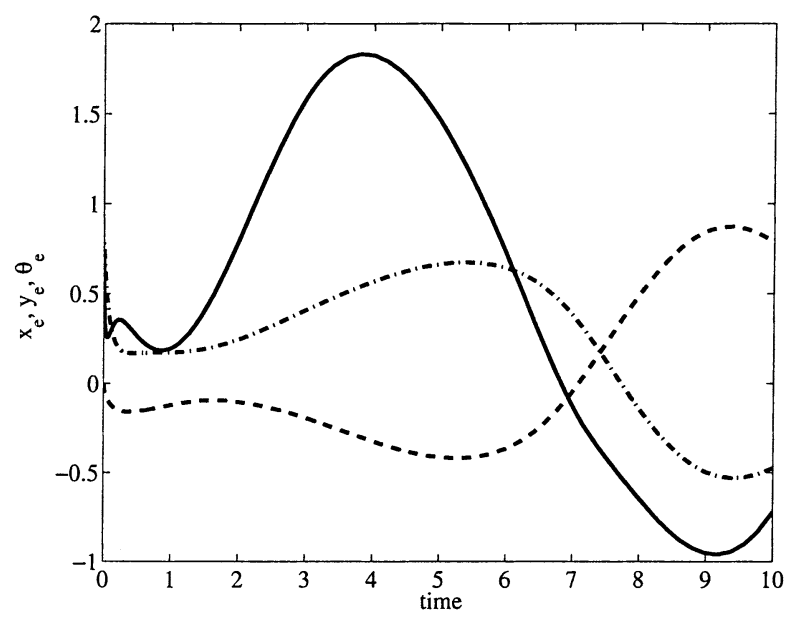

Fig. 5 Tracking errors without $\xi(\mathbf{x})$ (solid: $x_{e}$, dashed: $y_{e}$, dash$\left.\operatorname{dot}: \theta_{e}\right)$

that, by using the control law, the tracking error converges to zero in the case where there exists finite time $t_{d}$ such that $\tau_{d}(t)=0, \forall t \geq t_{d}$.

\section{Appendix A: The time derivative of $V(x)$}

The upper bound of time derivative of $V(x)$ along the solution trajectories of the system (18), (23) is given as follows.

$$
\begin{aligned}
\dot{V}= & \frac{\partial V}{\partial \mathbf{x}}[\mathbb{F}+\mathbb{G}(u+d)] \\
= & \frac{\partial V}{\partial \mathbf{x}}[\mathbb{F}+\mathbb{G} \Upsilon]+\frac{\partial V}{\partial \mathbf{x}} \mathbb{G}[-\xi+d] \\
\leq & -W(\mathbf{x})+z^{T}[-\xi+d] \\
= & -W(\mathbf{x})+\left[-\eta z_{1} \tanh \left(\kappa z_{1}\right)+z_{1} d_{1}\right] \\
& +\left[-\eta z_{2} \tanh \left(\kappa z_{2}\right)+z_{2} d_{2}\right]
\end{aligned}
$$

Firstly, let us consider the upper bound of the 2nd term of the right side of Eq. (36).

In the case of $z_{1} \geq 0$, the following inequality holds.

$$
-\eta z_{1} \tanh \left(\kappa z_{1}\right)+z_{1} d_{1} \leq \Xi_{1}^{+}\left(z_{1}\right), \forall z_{1} \geq 0
$$


where $\Xi_{1}^{+}\left(z_{1}\right) \triangleq-\eta z_{1} \tanh \left(\kappa z_{1}\right)+z_{1} \eta$. By taking the derivative of $\Xi_{1}^{+}\left(z_{1}\right)$ on $z_{1}$, we have

$$
\frac{d \Xi_{1}^{+}}{d z_{1}}=-\eta\left[\tanh \left(\kappa z_{1}\right)+\frac{\kappa z_{1}}{\cosh \left(\kappa z_{1}\right)^{2}}-1\right]
$$

From Eq. (38), it can be shown that $d \Xi_{1}^{+} / d z_{1}=0$ iff

$$
2 \kappa z_{1}-1=e^{-2 \kappa z_{1}}
$$

It is clear that Eq. (39) has a unique solution $z_{10}>0$. Then, taking the 2 nd derivative of $\Xi_{1}^{+}$on $z_{1}$ yields

$$
\frac{d^{2} \Xi_{1}^{+}}{d z_{1}^{2}}=-\eta\left[\frac{2 \kappa}{\cosh \left(\kappa z_{1}\right)^{2}}+\frac{2 \kappa^{2} z_{1} \sinh \left(\kappa z_{1}\right)}{\cosh \left(\kappa z_{1}\right)^{3}}\right]<0, \forall z_{1} \geq 0
$$

Equation (40) implies that $\Xi_{1}^{+}$is a concave function in $z_{1} \geq$ 0 . Hence, $\Xi_{1}^{+}$achieves the maximum value at $z_{1}=z_{10}$. Therefore, the following inequalities hold.

$$
\begin{aligned}
\Xi_{1}^{+}\left(z_{1}\right) & \leq \Xi_{1}^{+}\left(z_{10}\right) \\
& =\eta z_{10}\left[1-\tanh \left(\kappa z_{10}\right)\right] \\
& =\frac{2 \eta z_{10} e^{-2 \kappa z_{10}}}{1+e^{-2 \kappa z_{10}}} \\
& =\frac{\eta}{\kappa} e^{-2 \kappa z_{10}} \leq \frac{\eta}{\kappa}, \forall z_{1} \geq 0
\end{aligned}
$$

To derive the $3 \mathrm{rd}$ equality, we used $2 \kappa z_{10}-1=e^{-2 \kappa z_{10}}$.

Then let us consider the case of $z_{1} \leq 0$. In this case, the following inequality holds.

$$
-\eta z_{1} \tanh \left(\kappa z_{1}\right)+z_{1} d_{1} \leq \Xi_{1}^{-}\left(z_{1}\right), \forall z_{1} \leq 0
$$

where $\Xi_{1}^{-}\left(z_{1}\right) \triangleq-\eta z_{1} \tanh \left(\kappa z_{1}\right)-z_{1} \eta$. Then, based on the similar procedures in the case of $z_{1} \geq 0$, we can show that

$$
\Xi_{1}^{-}\left(z_{1}\right) \leq \frac{\eta}{\kappa}, \forall z_{1} \leq 0
$$

Therefore, from Eqs. (41) and (43), we obtain

$$
-\eta z_{1} \tanh \left(\kappa z_{1}\right)+z_{1} d_{1} \leq \frac{\eta}{\kappa}, \quad \forall z_{1} \in \mathcal{R}
$$

Similarly, we can show that

$$
-\eta z_{2} \tanh \left(\kappa z_{2}\right)+z_{2} d_{2} \leq \frac{\eta}{\kappa}, \quad \forall z_{2} \in \mathcal{R}
$$

Hence, from Eqs. (36), (44), (45), we obtain

$$
\dot{V} \leq-W(\mathrm{x})+\frac{2 \eta}{\kappa}, \forall \mathrm{x} \in \mathcal{R}^{5}
$$

\section{Appendix B: Proof of Eq. (34)}

Since the control system considered in this paper is time invariant, in the following, we assume that $t_{d}=0$. Clearly, $x(0)$ belongs to a bounded set. In the case where $\tau_{d}(t)=0, \forall t \geq 0$, the time derivative of $V(\mathbf{x})$ along the solution trajectories of the system (18), (23) is given as follows.

$$
\dot{V} \leq-W(\mathbf{x})-\eta z_{1} \tanh \left(\kappa z_{1}\right)-\eta z_{2} \tanh \left(\kappa z_{2}\right) \leq 0
$$

Hence, the closed-loop system (18), (23) is globally stable in the sense of Lyapunov and

$$
\bar{x}_{e}, y_{e}, \theta_{e}, \bar{v}, \bar{\omega} \in \mathcal{L}_{\infty}
$$

Then, from (17) and (48),

$$
\begin{aligned}
\left|\alpha_{\omega}\right| & \leq\left|\omega_{r}\right|+\gamma\left|y_{e} \| v_{r}\right|\left|\frac{\sin \theta_{e}}{\theta_{e}}\right|+c_{5} \gamma\left|\theta_{e}\right| \\
& \leq \omega_{r \max }+\gamma\left\|y_{e}\right\|_{\mathcal{L}_{\infty}} v_{r \max }+c_{5} \gamma\left\|\theta_{e}\right\|_{\mathcal{L}_{\infty}}
\end{aligned}
$$

Hence, from (15), (48) and (49), we can conclude that

$$
\alpha_{\omega} \in \mathcal{L}_{\infty}, \omega=\alpha_{\omega}+\bar{\omega} \in \mathcal{L}_{\infty}, x_{e}=\bar{x}_{e}+c_{3} \omega y_{e} \in \mathcal{L}_{\infty}
$$

By the way, by integrating the both sides of (47) over $[0, t]$, we obtain

$$
\begin{aligned}
0< & V(0)-c_{3} \int_{0}^{t} \omega^{2} y_{e}^{2} d t-c_{4} \int_{0}^{t} \bar{x}_{e}^{2} d t-c_{5} \int_{0}^{t} \theta_{e}^{2} d t \\
& -c_{6} \int_{0}^{t} \bar{v}^{2} d t-c_{7} \int_{0}^{t} \bar{\omega}^{2} d t-\eta \int_{0}^{t} z_{1} \tanh \left(\kappa z_{1}\right) d t \\
& -\eta \int_{0}^{t} z_{2} \tanh \left(\kappa z_{2}\right) d t
\end{aligned}
$$

Note that $z_{i} \tanh \left(\kappa z_{i}\right) \geq 0, \forall i=1,2$. Therefore, since the inequality (51) still holds even if $t \rightarrow \infty$, we get

$$
\omega^{2} y_{e}^{2}, \bar{x}_{e}^{2}, \theta_{e}^{2}, \bar{v}^{2}, \bar{\omega}^{2} \in \mathcal{L}_{1}
$$

Then we show (34) by using (48), (50), (52), Lemma 1 and Lemma 2.

- $\theta_{e}^{2}:$ From (18) and (48),

$$
\begin{aligned}
\frac{d}{d t}\left(\theta_{e}^{2}\right) & =2 \theta_{e} \dot{\theta}_{e} \\
& =2 \theta_{e}\left(-c_{5} \gamma \theta_{e}-\gamma y_{e} v_{r} \frac{\sin \theta_{e}}{\theta_{e}}-\bar{\omega}\right) \in \mathcal{L}_{\infty}
\end{aligned}
$$

Hence, with the help of Lemma 1, it follows that $\theta_{e} \rightarrow$ $0,(t \rightarrow \infty)$.

- $\bar{x}_{e}^{2}$ : From (18), (48) and (50),

$$
\begin{aligned}
\frac{d}{d t}\left(\bar{x}_{e}^{2}\right) & =2 \bar{x}_{e} \dot{\bar{x}}_{e} \\
& =2 \bar{x}_{e}\left(\omega y_{e}-c_{4} \bar{x}_{e}-\bar{v}\right) \in \mathcal{L}_{\infty}
\end{aligned}
$$

Hence, with the help of Lemma 1, it follows that $\bar{x}_{e} \rightarrow$ $0,(t \rightarrow \infty)$.

- $\quad \omega^{2} y_{e}^{2}$ : From (18), (48) and (50),

$$
\dot{y}_{e}=-c_{3} \omega^{2} y_{e}-\omega \bar{x}_{e}+v_{r} \sin \theta_{e} \in \mathcal{L}_{\infty}
$$

Thus,

$$
\begin{aligned}
\dot{\alpha}_{\omega}= & \dot{\omega}_{r}+\gamma \dot{y}_{e} v_{r} \frac{\sin \theta_{e}}{\theta_{e}}+\gamma y_{e} \dot{v}_{r} \frac{\sin \theta_{e}}{\theta_{e}}+\gamma y_{e} v_{r} \frac{d}{d t}\left(\frac{\sin \theta_{e}}{\theta_{e}}\right) \\
& +c_{5} \gamma \dot{\theta}_{e} \in \mathcal{L}_{\infty}
\end{aligned}
$$

By the way, from the definition, $\left\|\xi_{2}\right\|_{\mathcal{L}_{\infty}} \leq \eta$. Hence,

$$
\begin{aligned}
\dot{\omega} & =\dot{\bar{\omega}}+\dot{\alpha}_{\omega} \\
& =\left(-\dot{\alpha}_{\omega}+u_{2}\right)+\dot{\alpha}_{\omega} \\
& =\frac{1}{\gamma} \theta_{e}+\dot{\alpha}_{\omega}-c_{7} \bar{\omega}-\xi_{2} \in \mathcal{L}_{\infty}
\end{aligned}
$$

Thus,

$$
\frac{d}{d t}\left(\omega^{2} y_{e}^{2}\right)=2 \omega \dot{\omega} y_{e}^{2}+2 \omega^{2} y_{e} \dot{y}_{e} \in \mathcal{L}_{\infty}
$$

Hence, with the help of Lemma 1, it follows that $\omega y_{e} \rightarrow$ $0,(t \rightarrow \infty)$. 
- $x_{e}$ : From (15),

$x_{e}=\bar{x}_{e}+c_{3} \omega y_{e} \rightarrow 0,(t \rightarrow \infty)$

- $y_{e}$ :

- In the case where $v_{r}$ tends to 0 but $\omega_{r}$ does not converge to 0 .

$$
\begin{aligned}
\frac{d}{d t}\left(\bar{\omega}^{2}\right) & =2 \bar{\omega} \dot{\bar{\omega}} \\
& =2\left(\omega-\alpha_{\omega}\right)\left(-\dot{\alpha}_{\omega}+u_{2}\right) \\
& =2\left(\omega-\alpha_{\omega}\right)\left(\frac{1}{\gamma} \theta_{e}-c_{7} \bar{\omega}+\xi_{2}\right) \in \mathcal{L}_{\infty}
\end{aligned}
$$

Therefore, $\bar{\omega} \rightarrow 0,(t \rightarrow \infty)$. Hence,

$$
\begin{aligned}
\omega & =\bar{\omega}+\alpha_{\omega} \\
& =\bar{\omega}+\left(\omega_{r}+\gamma y_{e} v_{r} \frac{\sin \theta_{e}}{\theta_{e}}+c_{5} \gamma \theta_{e}\right) \rightarrow \omega_{r},(t \rightarrow \infty)
\end{aligned}
$$

From the fact that $\omega y_{e} \rightarrow 0,(t \rightarrow \infty)$, we can conclude that $y_{e} \rightarrow 0,(t \rightarrow \infty)$.

- In the case where $v_{r}$ does not converge to 0

From (18),

$$
\dot{\theta}_{e}=-c_{5} \gamma \theta_{e}+p(t)
$$

where

$$
p(t) \triangleq-\gamma y_{e} v_{r} \frac{\sin \theta_{e}}{\theta_{e}}-\bar{\omega}
$$

It can easily be verified that $p(t) \in \mathcal{L}_{\infty}$ and

$$
\begin{aligned}
\dot{p}(t)= & -\gamma \dot{y}_{e} v_{r} \frac{\sin \theta_{e}}{\theta_{e}}-\gamma y_{e} \dot{v}_{r} \frac{\sin \theta_{e}}{\theta_{e}}-\gamma y_{e} v_{r} \frac{d}{d t}\left(\frac{\sin \theta_{e}}{\theta_{e}}\right) \\
& -\dot{\bar{\omega}} \in \mathcal{L}_{\infty}
\end{aligned}
$$

Hence, form Lemma 2, $p(t) \rightarrow 0,(t \rightarrow \infty)$. Since $v_{r}(t)$ does not converge to $0, y_{e}(t) \rightarrow 0,(t \rightarrow \infty)$.

- $\omega-\omega_{r}$ :

Firstly, we show that $\bar{\omega}$ converges to 0 as $t \rightarrow \infty$. From

(18) and (23), we get

$$
\begin{aligned}
\frac{d}{d t} \bar{\omega}^{2} & =2 \bar{\omega} \dot{\bar{\omega}} \\
& =2 \bar{\omega}\left(-\dot{\alpha}_{\omega}+u_{2}\right) \\
& =2 \bar{\omega}\left(\frac{1}{\gamma} \theta_{e}-c_{7} \bar{\omega}-\xi_{2}\right) \in \mathcal{L}_{\infty}
\end{aligned}
$$

Moreover, from (52), $\bar{\omega}^{2} \in \mathcal{L}_{1}$. Hence, with the help of Lemma 1, it follows that $\bar{\omega} \rightarrow 0,(t \rightarrow \infty)$. Therefore, since $y_{e} \rightarrow 0,(t \rightarrow \infty)$, we can conclude that

$$
\begin{aligned}
\omega-\omega_{r} & =\left(\bar{\omega}+\alpha_{\omega}\right)-\omega_{r} \\
& =\bar{\omega}+\gamma y_{e} v_{r} \frac{\sin \theta_{e}}{\theta_{e}}+c_{5} \gamma \theta_{e} \rightarrow 0,(t \rightarrow \infty)
\end{aligned}
$$

- $v-v_{r}$ :

Firstly, we show that the boundedness of $\dot{\theta}_{e}, \dot{\alpha}_{\omega}, \dot{\omega}$. From (18), we get

$$
\dot{\theta}_{e}=-c_{5} \gamma \theta_{e}-\gamma y_{e} v_{r} \frac{\sin \theta_{e}}{\theta_{e}}-\bar{\omega} \in \mathcal{L}_{\infty}
$$

From (66) and (17), we have

$$
\begin{aligned}
\dot{\alpha}_{\omega}= & \dot{\omega}_{r}+\gamma \dot{y}_{e} v_{r} \frac{\sin \theta_{e}}{\theta_{e}}+\gamma y_{e} \dot{v}_{r} \frac{\sin \theta_{e}}{\theta_{e}}+\gamma y_{e} v_{r} \frac{d}{d t}\left(\frac{\sin \theta_{e}}{\theta_{e}}\right) \\
& +c_{5} \gamma \dot{\theta}_{e} \in \mathcal{L}_{\infty}
\end{aligned}
$$

Here, we used the fact that $\frac{d}{d t}\left(\frac{\sin \theta_{e}}{\theta_{e}}\right)=\frac{\partial}{\partial \theta_{e}}\left(\frac{\sin \theta_{e}}{\theta_{e}}\right) \dot{\theta}_{e} \in$ $\mathcal{L}_{\infty}$. Therefore,

$$
\begin{aligned}
\dot{\omega} & =\dot{\bar{\omega}}+\dot{\alpha}_{\omega} \\
& =\frac{1}{\gamma} \theta_{e}+\dot{\alpha}_{\omega}-c_{7} \bar{\omega}+\xi_{2} \in \mathcal{L}_{\infty}
\end{aligned}
$$

By the way,

$$
\begin{aligned}
\frac{d}{d t} \bar{v}^{2} & =2 \bar{v} \dot{\bar{v}} \\
& =2 \bar{v}\left(-\dot{\alpha}_{v}+u_{1}\right) \\
& =2 \bar{v}\left(\bar{x}_{e}-c_{6} \bar{v}+\xi_{1}\right) \in \mathcal{L}_{\infty}
\end{aligned}
$$

Hence, with the help of Lemma 1, it follows that $\bar{v} \rightarrow$ $0,(t \rightarrow \infty)$. From the above results, we can conclude that

$$
\begin{aligned}
v-v_{r}= & \left(\bar{v}+\alpha_{v}\right)-v_{r} \\
= & \bar{v}+v_{r} \cos \theta_{e}-c_{3} \dot{\omega} y_{e}+c_{3} \omega\left(\omega x_{e}-v_{r} \sin \theta_{e}\right) \\
& +c_{4} \bar{x}_{e}-v_{r} \rightarrow 0,(t \rightarrow \infty)
\end{aligned}
$$

\section{Appendix C: Lemma}

Lemma 1 (Barbălat $\left.{ }^{(8)}\right)$ If $\phi: \mathcal{R}_{+} \rightarrow \mathcal{R}$ is uniformly continuous and if the limit of the integral $\int_{0}^{t} \phi(\tau) d \tau$ exists as $t \rightarrow \infty$ and is finite, then $\lim _{t \rightarrow \infty} \phi(t)=0$.

Lemma $2{ }^{(6)}$ Consider a scalar system

$$
\dot{x}=-c x+p(t)
$$

where $c>0$ and $p(t)$ is a bounded and uniformly continuous function. If, for any initial time $t_{0} \geq 0$ and any initial condition $x\left(t_{0}\right)$, the solution $x(t)$ is bounded and converges to 0 as $t \rightarrow \infty$, then $\lim _{t \rightarrow \infty} p(t)=0$.

\section{Appendix D: The boundedness of the original state trajectory}

In this section, we show that the original state trajectory, namely, $\left[x_{e}, y_{e}, \theta_{e}, v, \omega\right]^{T}$ is bounded under the conditions of Theorem 1. From Theorem 1, $\bar{x}_{e}, y_{e}, \theta_{e}, \bar{v}, \bar{\omega} \in \mathcal{L}_{\infty}$. Hence, $\omega=\bar{\omega}+\omega_{r}+\gamma y_{e} v_{r} \sin \theta_{e} / \theta_{e}+c_{5} \gamma \theta_{e} \in \mathcal{L}_{\infty}$ and $x_{e}=$ $\bar{x}_{e}+c_{3} \omega y_{e} \in \mathcal{L}_{\infty}$. Further, $\dot{y}_{e}=-c_{3} \omega^{2} y_{e}-\omega \bar{x}_{e}+v_{r} \sin \theta_{e} \in \mathcal{L}_{\infty}$ and $\dot{\theta}_{e}=-c_{5} \gamma \theta_{e}-\gamma y_{e} v_{r} \sin \theta_{e} / \theta_{e}-\bar{\omega} \in \mathcal{L}_{\infty}$. From these facts, it can be shown that $\dot{\alpha}_{\omega} \in \mathcal{L}_{\infty}$. Hence, $\dot{\omega}=\theta_{e} / \gamma+\dot{\alpha}_{\omega}-c_{7} \bar{\omega}+$ $d_{2} \in \mathcal{L}_{\infty}$. Hence, $v=\bar{v}+c_{4} \bar{x}_{e}+v_{r} \cos \theta_{e}-c_{3} \dot{\omega} y_{e}+c_{3} \omega\left[\omega x_{e}-\right.$ $\left.v_{r} \sin \theta_{e}\right] \in \mathcal{L}_{\infty}$.

\section{Appendix E: The boundedness of $u(x)$}

In this section, we show that the boundedness of $u(\mathrm{x})$ is guaranteed under the conditions of Theorem 1 and several additional conditions, namely, $\ddot{v}_{r}, \ddot{\omega}_{r}, \dot{\tau}_{d 3} \in \mathcal{L}_{\infty}$. Firstly, we show the boundedness of $u_{1}$. From Theorem 1 and Appendix D, $\dot{\bar{x}}_{e}=\omega y_{e}-c_{4} \bar{x}_{e}-\bar{v} \in \mathcal{L}_{\infty}$ and $\dot{x}_{e}=\dot{\bar{x}}_{e}+c_{3} \dot{\omega} y_{e}+$ 
$c_{3} \omega \dot{y}_{e} \in \mathcal{L}_{\infty}$ and $\dot{\bar{\omega}}=\theta_{e} / \gamma-c_{7} \bar{\omega}-\xi_{2}+d_{2} \in \mathcal{L}_{\infty}$. Note that, from Assumption $1, d_{2}=-\tau_{d 3} / J \in \mathcal{L}_{\infty}$. Based on these results, we can show that $\ddot{y}_{e}, \ddot{\theta}_{e} \in \mathcal{L}_{\infty}$. Moreover, by using the above results and the additional assumptions on the reference trajectories, namely, $\ddot{v}_{r}, \ddot{\omega}_{r} \in \mathcal{L}_{\infty}$, we can show that $\ddot{\alpha}_{\omega} \in \mathcal{L}_{\infty}$. Hence, $\ddot{\omega}=\dot{\theta}_{e} / \gamma+\ddot{\alpha}_{\omega}-c_{7} \dot{\omega}+\dot{\xi}_{2}+\dot{d}_{2} \in \mathcal{L}_{\infty}$. Note that we used $\dot{d}_{2}=-\dot{\tau}_{d 3} / J \in \mathcal{L}_{\infty}$. Therefore, we can show $\dot{\alpha}_{v} \in \mathcal{L}_{\infty}$. Hence, we can conclude that $u_{1}=$ $\bar{x}_{e}+\dot{\alpha}_{v}-c_{6} \bar{v}+\xi_{1} \in \mathcal{L}_{\infty}$. Finally, we can show that $u_{2}$ is bounded, since $u_{2}=\theta_{e} / \gamma+\dot{\alpha}_{\omega}-c_{7} \bar{\omega}+\xi_{2} \in \mathcal{L}_{\infty}$ from Eq. (23).

\section{References}

( 1 ) Aguilar, L.E., Souères, M.P., Courdesses, M. and Fleury, S., Robust Path-Following Control with Exponential Stability for Mobile Robots, IEEE Int. Conf. Robotics \& Automation, (1998), pp.3279-3284.

( 2 ) Canudas de Wit, C., Siciliano, B. and Bastineds, G., Theory of Robot Control, (1996), Springer-Verlag.

( 3 ) Dixon, W.E., Dawson, D.M., Zergeroglu, E. and Zhang, F., Robust Tracking and Regulation Control for
Mobile Robots, Int. J. Robust \& Nonlinear Control, Vol.10 (2000), pp.199-216.

( 4 ) Fierro, R. and Lewis, F.L., Control of a Nonholonomic Mobile Robot: Backstepping Kinematics into Dynamics, Proc. IEEE Conf. Decision \& Control, (1995), pp.3805-3810.

( 5 ) Fukao, T., Nakagawa, H. and Adachi, N., Adaptive Tracking Control of a Nonholonomic Mobile Robot, IEEE Trans. Robotics \& Automation, Vol.16, No.5 (2000), pp.609-615.

( 6 ) Jiang, Z. and Nijmeijer, H., Tracking Control of Mobile Robots: A Case Study in Backstepping, Automatica, Vol.33, No.7 (1997), pp.1393-1399.

( 7 ) Kanayama, Y., Kimura, Y., Miyazaki, F. and Noguchi, T., A Stable Tracking Control Method for an Autonomous Mobile Robot, Proc. IEEE Int. Conf. Robotics \& Automation, (1990), pp.384-389.

( 8 ) Khalil, H., Nonlinear Systems, (1996), Prentice-Hall.

( 9 ) Qu, Z. and Dawson, D.M., Robust Tracking Control of Robot Manipulators, (1996), IEEE Press. 